\title{
Performance of the gamma-ray camera based on scintillator array and PSPMT with an ASIC readout system
}

\author{
Kazuki Ueno ${ }^{* a}$, Kaori Hattori ${ }^{a}$, Chihiro Ida ${ }^{a}$, Satoru Iwaki ${ }^{a}$, Shigeto Kabuki ${ }^{a}$, \\ Hidetoshi Kubo $^{a}$, Shunsuke Kurosawa ${ }^{a}$, Kentaro Miuchi $^{a}$, Hironobu Nishimura ${ }^{a}$, \\ Atsushi Takada ${ }^{a}$ Toru Tanimori $^{a}$, and Hiroyuki Sekiya ${ }^{b}$ \\ ${ }^{a}$ Kyoto University \\ ${ }^{b}$ ICRR \\ E-mail: kazuki@cr.scphys.kyoto-u.ac.jp
}

\begin{abstract}
We have studied the performance of a readout system with ASIC chips for our gamma camera based on 64 channels multi-anode PSPMT(Hamamatsu flat-panel H8500) coupled to a GSO(Ce) scintillator array. The GSO array consists of $8 \times 8$ pixels of $6 \times 6 \times 13 \mathrm{~mm}^{3}$ with the same pixel pitch as the anode of H8500. This camera is intended for an absorber of an Electron Tracking Compton gamma-ray camera which measures up to about $1 \mathrm{MeV}$ gamma-ray. We need a readout system with low power consumption in order to make a camera which has $64 \times 108$ readout channels for a balloon-borne experiment. We adopted the ASIC chip, IDEAS VA32_HDR11, with low power consumption of $1.3 \mathrm{~W} / 64 \mathrm{ch}$ and dynamic range of $\sim-35 \mathrm{pC}$, which is one of chips with widest range among commercial ones. However, we have to operate the H8500 with the low gain of about $10^{5}$ because it only measures up to about $100 \mathrm{keV}$ gamma-ray with the typical gain of about $10^{6}$, and we developed an attenuator board in order to operate the H8500 with the typical gain. The attenuator board has 64 singular resistors adjusted to the anode gain and uniforms variation of the anode gain of the H8500, min:max 1:2.3, before inputs to ASIC chips. Using the board, The H8500 was able to operate with the gain of about $10^{6}$ and the output signals from that board had a good uniformity of min:max $\sim 1: 1.2$. The system with the new attenuator board has the incident energy dynamic range of 30 to $900 \mathrm{keV}$, the position resolution of less than $6 \mathrm{~mm}$, and a typical energy resolution of about $11.7 \%$ (FWHM) at $662 \mathrm{keV}$.
\end{abstract}

International workshop on new photon-detectors PD07

June 27-29 2007

Kobe University, Kobe, Japan

\footnotetext{
${ }^{*}$ Speaker.
} 


\section{Introduction}

We have been developing an Electron Tracking Compton Camera for Sub-MeV to MeV gamma ray astronomy[1], especially for a balloon experiment. The camera needs a scintillation camera as an absorber for Compton-scattered gamma rays, which has good energy and position resolutions, the wide dynamic range, and a large size. Their resolutions, the range, and the efficiency of Compton-scattered gamma rays contribute to the angular resolution, the range, and the efficiency of the Compton Camera. For these requirements, we use the scintillation camera which consists of a position sensitive photomultiplier (PSPMT) and a pixelated scintillator array (PSA). We adopt the Hamamatsu 64 channels multi-anode PSPMT H8500[2] and a $\mathrm{GSO}(\mathrm{Ce})\left(\mathrm{Gd}_{2} \mathrm{SiO}_{5}\right.$ : Ce) crystal array which fits to the anode pitches of the $\mathrm{H} 8500$ as PSA[3]. The H8500 was recently developed as the application to nuclear physics and medicine, for example, Cherenkov detector, PET, and SPECT $[4,5,6,7]$. It has the advantage such as a much smaller dead space and a larger effective area (89\% of the package size) than previous multi-anode PMTs. GSO $(\mathrm{Ce})$ has advantages in astronomical use such as greater radiation hardness than most of known scintillators. Also, It can be easily processed because it is nonhydroscopic.

In addition, a readout system with low power consumption $(\leq 2 \mathrm{~W} / 64 \mathrm{ch})$ for our scintillation camera is required because the power is limited in the sky for the balloon experiment. In order to satisfy the requirement, we chose the readout system of scintillation camera with the ASIC chips, IDEAS VA32_HDR11, with low power consumption of $1.3 \mathrm{~W} / 64 \mathrm{ch}$ and dynamic range of $35 \mathrm{pC}$, which is one of chips with widest range among commercial ones. However, we have to operate the $\mathrm{H} 8500$ with the low gain of about $10^{5}$ because it only measures up to about $100 \mathrm{keV}$ gamma-ray with the typical gain of about $10^{6}$. We developed an attenuator board in order to operate the H8500 with the typical gain, which can measure up to about $1 \mathrm{MeV}$ gamma-ray.

In this paper, we report the performances of the readout system of the scintillation camera with commercial ASIC chips and the attenuator board developed by us.

\section{Scintillation Camera}

The H8500 has the compact size of $52 \mathrm{~mm} \times 52 \mathrm{~mm} \times 28 \mathrm{~mm}$ with 12 stage metal channel anodes. The active photocathode area is $49 \mathrm{~mm} \times 49 \mathrm{~mm}$ with a matrix of $8 \times 8$ anodes. Each anode pixel size is $5.8 \mathrm{~mm} \times 5.8 \mathrm{~mm}$ and the pitch between center of the anodes is $6.08 \mathrm{~mm}$. The typical anode gain is about $10^{6}$ under a High Voltage (HV) of $-1000 \mathrm{~V}$ and the typical anode gain uniformity (the ratio of the maximum gain to the minimum gain) is about 3.0.

The GSO(Ce) PSA consists of $8 \times 8$ pixels of $6 \times 6 \times 13 \mathrm{~mm}^{3}$ with the same pixel pitch as the anode of H8500. The 64 pixels are optically isolated by Vikuti 3M ESR which is a multilayer polymer mirror with a thickness of $65 \mu \mathrm{m}$ and a reflectance of $98 \%$. The PSA is glued to the H8500 with OKEN-6262A optical grease. Figure.1 shows the picture of our Scintillation Camera.

\section{Readout system}

\subsection{CP80068 system}

Figure.2 shows the individual anode readout system Clear Pulse Co. Ltd. CP80068. It is 


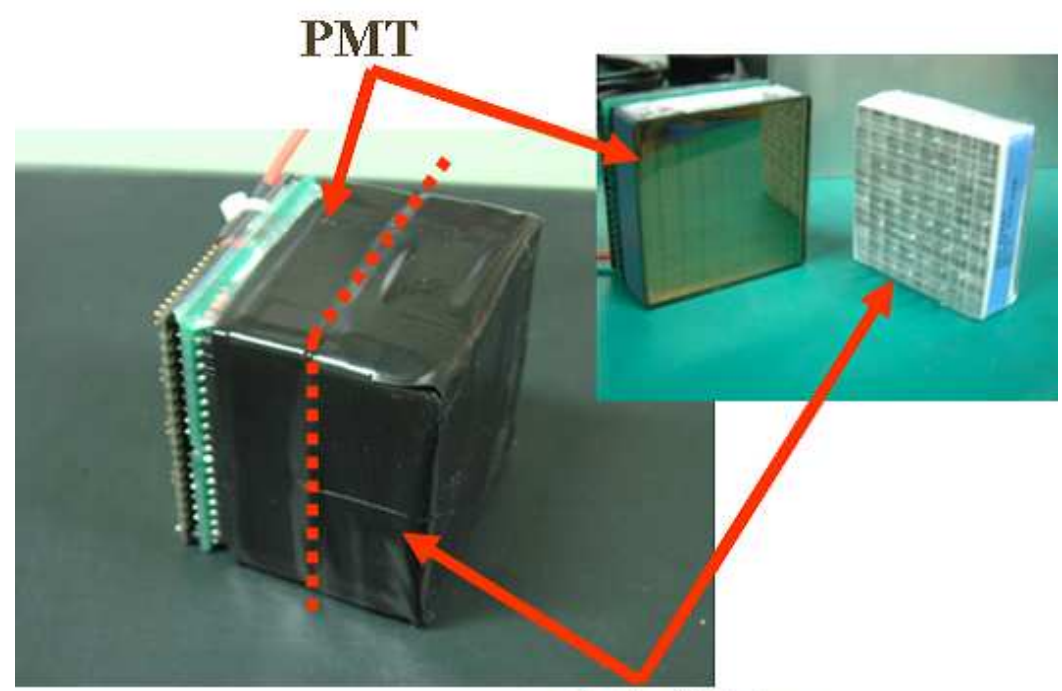

Scintillator

Figure 1: Picture of GSO(Ce) PSA with the H8500.

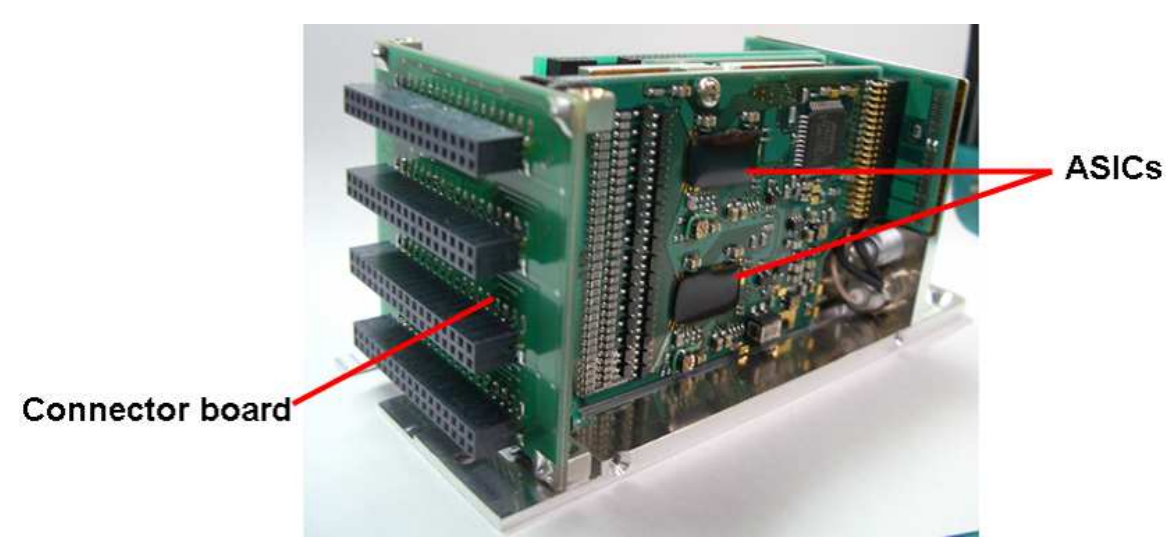

Figure 2: Picture of CP80068. CP80068 consists of the board containing two VA32_HDR11 chips and two TA32CG2 chips, and the connector board which connects ASIC chips to the H8500. The connector board is replaceable.

designed for two dimensional array of the H8500 and has the size of $52 \mathrm{~mm} \times 52 \mathrm{~mm} \times 95 \mathrm{~mm}$. CP80068 is based on two types of analog ASICs, 32 channel VA32_HDR11 and TA32CG2 manufactured by IDEAS ASA and consists of two VA32_HDR11 chips and two TA32CG2 chips. The VA32_HDR11 contains pre-amplifiers with input dynamic range of about $20 \mathrm{pC}$ to $35 \mathrm{pC}$, shapers with gain of $118 \mathrm{mV} / \mathrm{pC}$ and peaking time of $0.7 \mu \mathrm{s}$, sample and hold circuits and a multiplexer. The TA32CG2 contains fast shapers with peaking time of $75 \mathrm{~ns}$ and discriminators, which make the trigger signals. Figure. 3 shows a schematic diagram of the data acquisition system. The multiplexed 64 channels data are digitized by a flash ADC on the CP80068 and sent to the VME sequence module via FPGAs. It takes $164 \mu$ s to read 64 channels. 


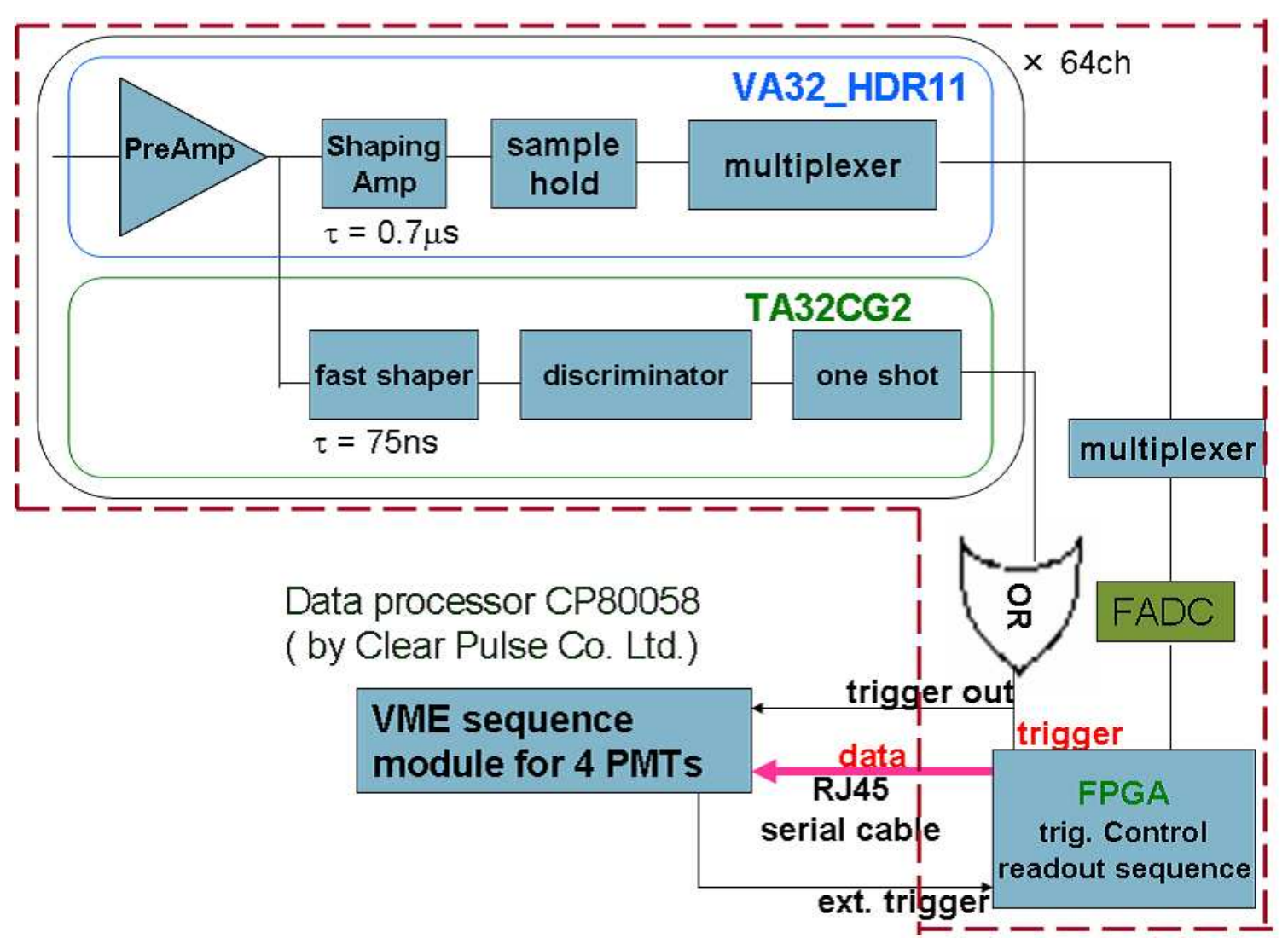

Figure 3: Schematic view of data acquisition system.

\subsection{Attenuator board}

The VA32_HDR11 has the narrow dynamic range corresponding to that we have to operate the $\mathrm{H} 8500$ with the low gain of about $10^{5}$. It was reported, if the $\mathrm{H} 8500$ is operated with the low gain, the energy dynamic range to the incident gamma rays and the energy resolution of scintillation camera are narrow and bad, respectively, compared to the operation of the H8500 with the typical gain[8]. In order to resolve their problems, we developed the attenuator board. The concept of the attenuator board is as follows: To obtain the good energy resolution of scintillation camera, signals get the gain of about $10^{6}$ in the H8500. Because of the narrow dynamic range of VA32_HDR11, obtained signals are attenuated within the range before VA32_HDR11. In addition, considering the anode gain variation of the H8500, those signals are uniformed before VA32_HDR11. On the basis of the concept, we made the attenuator board as shown in the Figure.4, which has 64 singular resistors adjusted to the anode gain and uniforms variation of the anode gain of $\mathrm{H} 8500$ before VA32_HDR11. Because the board is as the same size as the readout board of CP80068 (Figure.4), we only replace the connector board by the attenuator board and the total size of CP80068 and Scintillation camera dose not change.

\section{Measurement and results}

As described above, we are interested in the sub-MeV gamma-ray region. $662 \mathrm{keV}$ gamma 


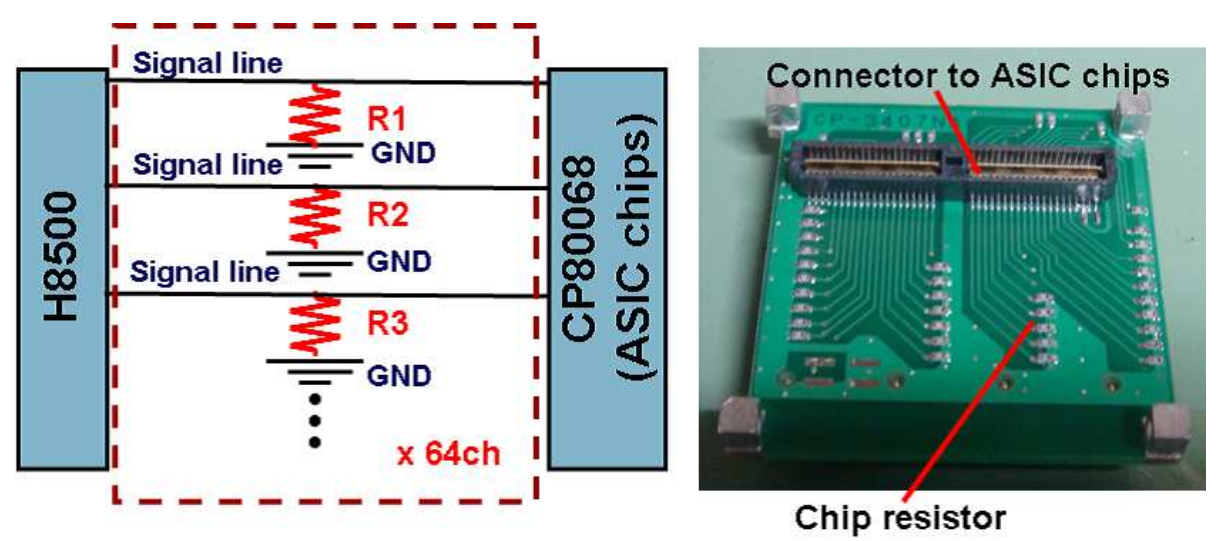

Figure 4: Schematic view of the attenuator board(left, dashed line) and picture of the attenuator board(right). The readout board (Figure.2) can be replaced with the attenuator board.

rays $\left(1 \mathrm{MBq}{ }^{137} \mathrm{Cs}\right.$ source) were irradiated to the $\mathrm{GSO}(\mathrm{Ce}) \mathrm{PSA}$ at a distance of $30 \mathrm{~cm}$. For the energy calibration, ${ }^{54} \mathrm{Mn}(835 \mathrm{keV}),{ }^{57} \mathrm{Co}(122 \mathrm{keV})$, and ${ }^{133} \mathrm{Ba}(31,81,356 \mathrm{keV})$ were also used. H8500 was operated with the gain of about $10^{6}(\mathrm{HV} \sim-900 \mathrm{~V})$.

The $662 \mathrm{keV}$ peak values of 64 channels are shown in Figure.5. The measured minimum gain to maximum gain ratio with the attenuator board is $\sim 1: 1.2$. We investigated the relation between resistance and attenuation factor. Considering that relation, we chose not variable but discrete resistance of commercial resistor on the attenuator board in order to make the compact board, and we expected that the ratio is $1: 1.1 \pm 0.1$. The result is in agreement with the expectation.

The obtained flood irradiation image of ${ }^{137} \mathrm{Cs}$ are shown in Figure.6. The position of the hit point of the $\mathrm{GSO}(\mathrm{Ce}) \mathrm{PSA}$ is reconstructed to calculate the center of gravity of 64 channels ADC value using the following equations,

$$
x=\frac{\sum_{i=1}^{64} P_{i} \cdot(i \bmod 8)}{\sum_{i=1}^{64} P_{i}} \quad y=\frac{\sum_{i=1}^{64} P_{i} \cdot(i \operatorname{div} 8)}{\sum_{i=1}^{64} P_{i}}
$$

where $\mathrm{P}_{i}$ is the ADC output of $i$ th anode of CP80068 system. The peak to valley ratio of the $\mathrm{x}$ projection of the flood irradiation image as shown in Figure.6 is good. It is shown that each 64 pixel is clearly resolved and that the position resolution is less than a pixel pitch of $6 \mathrm{~mm}$. We also obtained the energy spectrum of each pixel. The typical energy spectrum is shown in Figure.6 and the typical energy resolution is $11.7 \%$ (FWHM) @ $662 \mathrm{keV}$. Figure.7 shows the energy spectra and the energy resolutions of measured energy from other sources. Those resolution are fit to $\frac{\Delta E}{E}=11.7\left(\frac{E}{662[\mathrm{keV}]}\right)^{-0.49}$ in the range of $31 \mathrm{keV}$ to $835 \mathrm{keV}$. This means that the measurable energy dynamic range of the system is about 30 to $900 \mathrm{keV}$ at all 64 pixels.

In addition, the energy resolution and the energy dynamic range of the system without the attenuator board was measured. The typical energy resolution is 13.0\% (FWHM) @ $662 \mathrm{keV}$ and the energy dynamic range is about 100 to $700 \mathrm{keV}$ at all pixels with the $\mathrm{H} 8500$ gain of $10^{5}$. Those results indicate that the system of the scintillation camera with the attenuator board has adequate performances. 


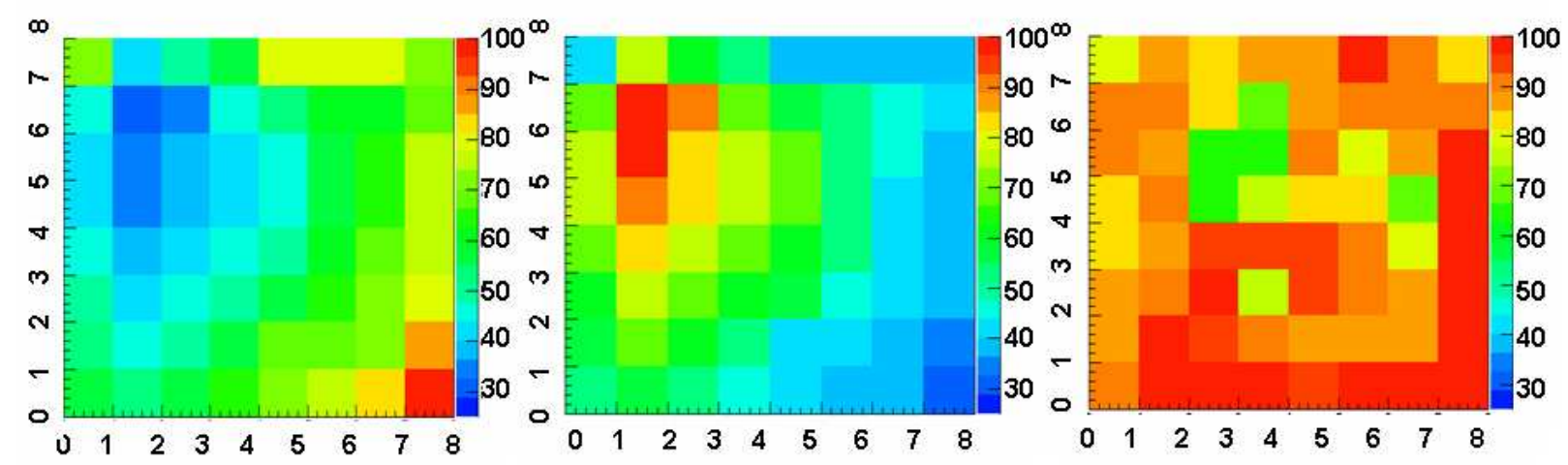

Figure 5: Anode gain uniformity map of the typical H8500 (left), resistance value map of the attenuator board we developed corresponding to the left one (center), and measured peak value map (right), where maximum peak value is set to100.
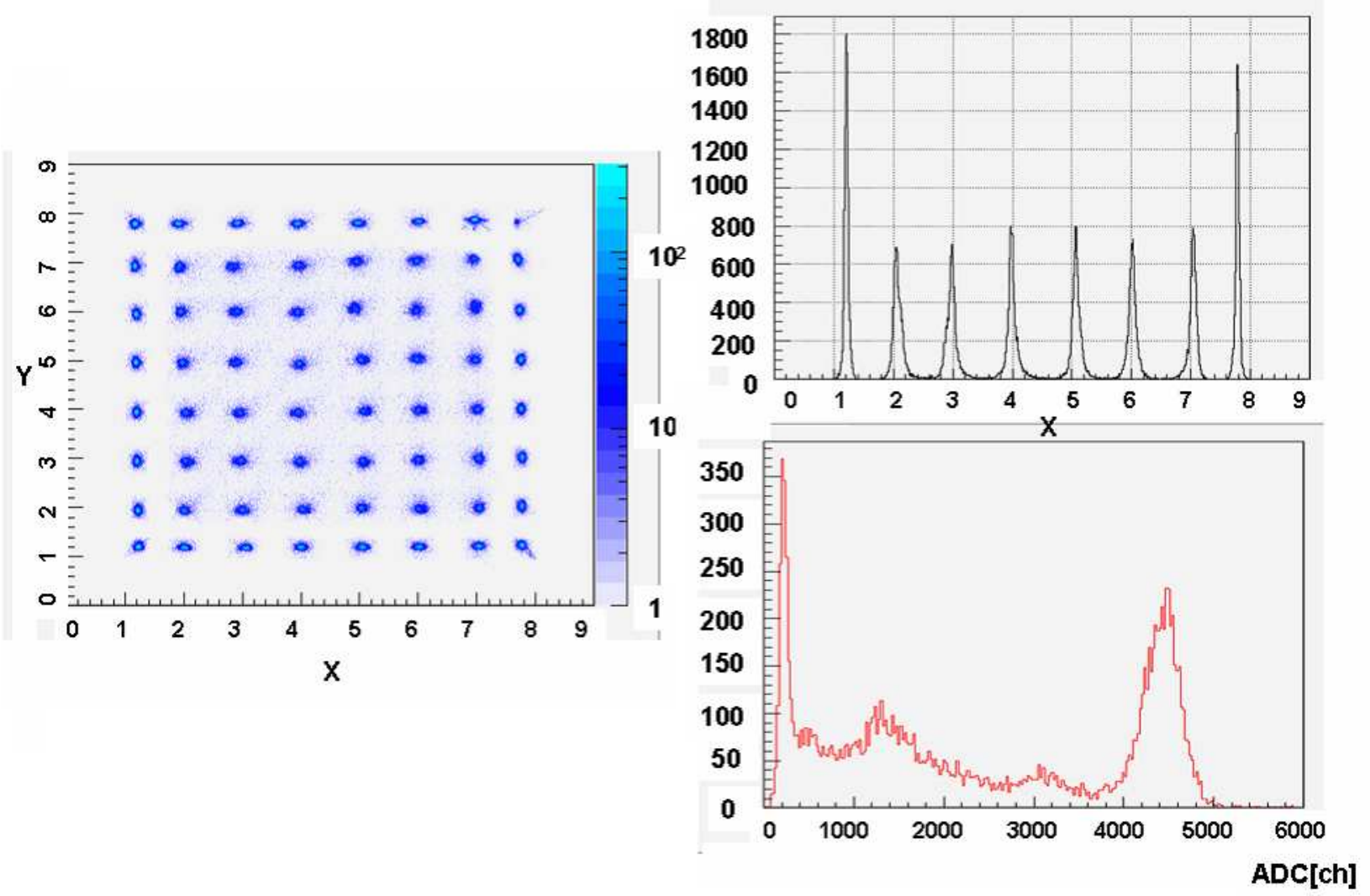

Figure 6: Flood field image of ${ }^{137} \mathrm{Cs}$ irradiation(left), $\mathrm{x}$-projection map of the left image at the 4th row $(3.5<Y<4.5)$ (upper right), and typical energy spectrum(lower right) 


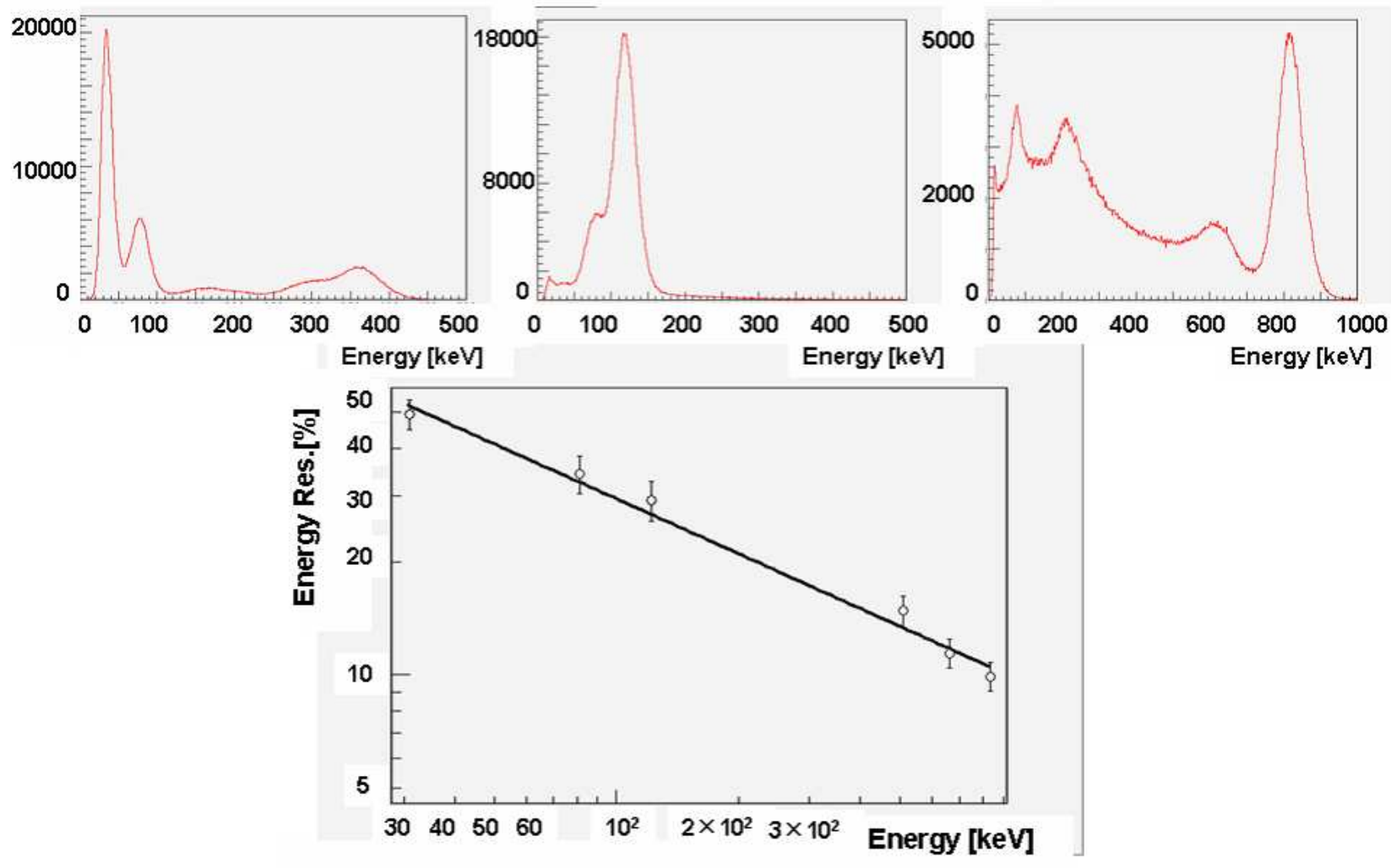

Figure 7: Measured energy spectra of ${ }^{133} \mathrm{Ba}$ (upper left), ${ }^{57} \mathrm{Co}$ (upper center), and ${ }^{54} \mathrm{Mn}$ (upper right), and measured typical energy resolution(bottom). The best fit line is described in the text.

\section{Summary}

We adopted the readout system of the scintillation camera with commercial ASIC and the attenuator board we developed and investigated the performances of the system. As a result, we obtained the good resolution and the wide energy dynamic range of the scintillation camera with low power consumption. The performance of the system is sufficient to use as a Compton-scattered gamma ray camera of our Compton camera for the balloon experiment. We have been constructing the Compton camera and testing its performance.

\section{Acknowledgments}

This work is supported by a Grant-in-Aid for the 21st Century COE "Center for Diversity and Universality in Physics"; a Grant-in-Aid in Scientific Research of the Japan Ministry of Education, Culture, Science, Sports, Technology; "SENTAN",JST.

\section{References}

[1] A. Takada, et al., Nucl. Instr. and Meth. A 546 (2005) 258. 
[2] Hamamatsu technical data sheet H8500-H8500B, February 2005, printed in Japan, (http://www.hamamatsu.com).

[3] H. Nishimura, et al., Nucl. Instr. and Meth. A 573 (2007) 115.

[4] T. Matsumoto, et al., Nucl. Instr. and Meth. A 521 (2004) 367.

[5] R. Pani, et al., Nucl. Instr. and Meth. A 527 (2004) 54;

R. Pani, et al., Nucl. Instr. and Meth. A 513 (2003) 36.

[6] M. Gimenez, et al., Nucl. Instr. and Meth. A 525 (2004) 298.

[7] D. Herbert, et al., Nucl. Instr. and Meth. A 518 (2004) 399.

[8] H. Sekiya, et al., Nucl. Instr. and Meth. A 563 (2006) 49. 